Research Article

\title{
Stepped Frequency Multiresolution Digital Signal Processing
}

\author{
Qunying Chen \\ Xi'an Peihua University, Xi'an, Shaanxi 710125, China \\ Correspondence should be addressed to Qunying Chen; 150444@peihua.edu.cn
}

Received 29 April 2021; Revised 26 May 2021; Accepted 31 May 2021; Published 9 June 2021

Academic Editor: Yi-Zhang Jiang

Copyright (c) 2021 Qunying Chen. This is an open access article distributed under the Creative Commons Attribution License, which permits unrestricted use, distribution, and reproduction in any medium, provided the original work is properly cited.

With the rapid development of radar industry technology, the corresponding signal processing technology becomes more and more complex. For the radar with short-range detection function, its corresponding signal mostly presents the characteristics of wide bandwidth and multiresolution. In the traditional data processing process, a large number of signals will interfere with the signal, which makes the final signal processing difficult or even impossible. Based on this problem, this paper proposes a principal component linear prediction processing algorithm based on clutter suppression processing on the basis of traditional signal processing algorithm. According to the curve characteristics of the data returned by the target detected by the signal, through certain image signal measurement and transformation, the clutter can be effectively suppressed and the typical characteristics of the corresponding target curve can be enhanced. For the convergence problem of signal processing and the corresponding image chromatic aberration compensation problem, this paper will realize the chromatic aberration compensation of the corresponding target echo image based on the radial pointing transverse mode algorithm and enhance the convergence speed of the whole algorithm system. In the experimental part of this paper, the optimization algorithm proposed in this paper is compared with the traditional algorithm. The experimental results show that the algorithm proposed in this paper has obvious advantages in the convergence of signal processing and antijamming performance and has the promotion value.

\section{Introduction}

With the rapid development of information technology and electronic technology, radar technology has been further developed. The range resolution of common low altitude detection radar mainly depends on the bandwidth of the transmitted signal, the corresponding broadband data signal processing technology and antijamming technology [1-3]. At present, the traditional stepped frequency multiresolution digital signal processing technology in low altitude radar detection technology mainly focuses on the theoretical level, and the efficient and reasonable digital signal processing technology is still the problem of low altitude radar signal processing $[4,5]$. At present, the factors that affect the signal echo of low altitude detection radar include the direct coupling of signals between antenna transceivers of low altitude detection radar, the large reflection on the ground and underground of the signal generating place, and a variety of irresistible reflection sources near the transmitting place, and the interference transmitting source mainly comes from the complex and huge DSP printed board. The corresponding data signal needs to be converted between different frequency domains when processing the corresponding data signal, which will cause the sidelobe blocking phenomenon between the corresponding signals, thus forming a strong interference [6-8].

The traditional stepped frequency multiresolution digital signal processing mainly focuses on the system hardware design optimization and echo signal optimization processing. In the corresponding hardware level, it is not the key problem to be considered in this paper. For the digital signal processing level, it mainly deals with the close range digital clutter. The corresponding traditional processing methods include average method digital signal processing algorithm, digital signal principal component analysis algorithm, and corresponding digital signal linear prediction algorithm [9-11]. In the corresponding digital signal average processing algorithm, it is limited by the detection environment, which requires the corresponding detection environment. If 
it is the ground, it requires the ground to be as smooth as possible. When the detection environment is low altitude, it requires less interference. After meeting the relevant environmental requirements, it averages the corresponding elements of the echo, so as to filter a large number of clutters. In essence, it is to arrange and combine a large number of original data returned by echo in a certain logical order and form a twodimensional sequence, corresponding to each element in the sequence minus the average value one by one [12]. The corresponding digital signal principal component analysis algorithm assumes that the clutter presents a Gaussian distribution during detection. Under this assumption, the signal is decomposed by wavelet, so as to reduce the clutter component of radar detection signal. For the principal component of digital signal, it is decomposed by two-dimensional determinant, and finally the related clutter is suppressed [13]. For the digital signal linear prediction processing algorithm, it mainly uses the autoregressive average algorithm to process. In the process of processing, it takes the related signal as the reference signal and predicts the expected output of other signals. Under the corresponding mathematical model, it transmits the corresponding reference signal to the input of the digital signal processing algorithm. In this way, we can maximize the correlation between the corresponding algorithm processing ability and the corresponding processing effect [14]. Based on the above situation, it can be seen that the traditional digital signal processing technology has some problems, especially in the convergence and anti-interference degree of the algorithm which can not achieve a certain compromise. Based on this, this paper proposes a principal component linear prediction processing algorithm based on clutter suppression processing according to the curve characteristics of the data returned by the target detected by the signal, the clutter can be effectively suppressed, and the typical characteristics of the corresponding target curve can be enhanced through certain image signal measurement and transformation. For the convergence problem of signal processing and the corresponding image chromatic aberration compensation problem, this paper will realize the chromatic aberration compensation of the corresponding target echo image based on the radial pointing transverse mode algorithm and enhance the convergence speed of the whole algorithm system. In the experimental part of this paper, the optimization algorithm proposed in this paper is compared with the traditional algorithm. The experimental results show that the algorithm proposed in this paper has obvious advantages in the convergence of signal processing and antijamming performance and has the promotion value.

The structure of this paper is as follows: in the second section, the current research status of stepped frequency multiresolution digital signal processing algorithm is analyzed and discussed. In the third section of this paper, we will focus on the analysis of stepped frequency multiresolution digital signal processing algorithm, mainly on the analysis of principal component linear prediction processing algorithm based on clutter suppression processing and radius pointing transverse mode algorithm. In the fourth section of this paper, we will make a comparative experiment on the algorithm. Finally, this paper is summarized.

\section{Related Work Analysis: Research Status of Stepped Frequency Multiresolution Digital Signal Processing Algorithm}

The main technical difficulty in the field of stepped frequency low altitude radar detection is digital signal processing algorithm. Stepped frequency radar mainly transmits a group of wideband pulse signals by carrier frequency hopping. The corresponding echo signal processing technology becomes the difficulty of digital signal processing $[15,16]$. Through the research and analysis of a large number of scientific research institutions and military research institutes, there are three main frequency step multiresolution digital signal processing algorithms, which are corresponding to the mean value method, digital signal principal component analysis algorithm, and corresponding digital signal linear prediction algorithm. The emphasis of the three algorithms is different, and the corresponding data signal processing technology has its own advantages and disadvantages. In the corresponding level of digital signal processing, it mainly includes digital signal average method, which is the so-called two-dimensional sequence processing algorithm, digital signal prediction algorithm, and other mainstream digital signal processing algorithms. The details of the corresponding algorithms are as follows: in the algorithm of digital signal average method, it is limited by the detection environment. If the detection environment is the ground, the ground should be as flat as possible. When the environment to be detected is low, less interference is needed. After meeting the relevant environmental requirements, the corresponding elements of the echo are averaged to filter out a large number of clutters. In essence, a large number of raw data returned by the echo are arranged and combined in a certain logical order to form a two-dimensional sequence. Each element in the corresponding sequence is subtracted from the average one by one. The algorithm has strong antijamming performance, but it has more strict requirements on the experimental environment, and most detection environments are complex and changeable [17-19]. The corresponding algorithm of digital signal principal component analysis assumes that the clutter presents Gaussian distribution in the corresponding detection. Under this assumption, the signal is decomposed by wavelet to reduce the clutter component of radar detection signal. The main components of digital signal are decomposed by two-dimensional determinant to suppress the correlated clutter. The algorithm has simple requirements for the environment and strong anticlutter processing ability, but the corresponding calculation is relatively complex, leading to serious convergence problems [20, 21]. For the linear prediction processing algorithm of digital signal, the autoregressive average algorithm is mainly used. In the process, the correlation signal is used as the reference signal to predict the expected output of other signals. In the corresponding mathematical model, the corresponding reference signal is transmitted to the input of digital signal processing algorithm, so as to maximize the correlation between the corresponding processing capacity and the 
corresponding processing effect. The algorithm still has the problem of convergence [22-24]. Therefore, on the basis of the above analysis, combined with the actual situation of low altitude detection processing, it is of great significance to optimize the frequency step multiresolution digital signal processing algorithm.

\section{Analysis and Research on Optimization Algorithm of Stepped Frequency Multiresolution Digital Signal Processing}

This section mainly analyzes and studies the stepped frequency multiresolution signal processing algorithm. The main core algorithms are the principal component linear prediction processing algorithm and the radius pointing transverse mode algorithm. The corresponding algorithm architecture is shown in Figure 1. The corresponding algorithm architecture can be seen from the figure, and the corresponding algorithm hardware module architecture is also shown in the corresponding architecture diagram. As shown in Figure 1, the core algorithm is mainly based on the traditional signal processing algorithm, and the principal component linear prediction processing algorithm based on clutter suppression processing is proposed. According to the curve characteristics of the data returned by the target detected by the signal, through certain image signal measurement and transformation, the clutter can be effectively suppressed, and the typical characteristics of the corresponding target curve can be enhanced. So it gets rid of the disadvantages of single mean algorithm, principal component algorithm, and linear prediction algorithm. For the convergence problem of signal processing and the corresponding image chromatic aberration compensation problem, this algorithm is mainly based on the radial pointing transverse mode algorithm to realize the chromatic aberration compensation of the corresponding target echo image and enhance the convergence speed of the whole algorithm system. In the corresponding hardware design level, this paper mainly discusses the selection and configuration circuit design of FPGA and DSP and discusses the related design of auxiliary circuit.

In the corresponding experimental level, this paper first carries out the accurate modeling of the echo data signal and then carries out the experiment based on the correlation modeling. In the experimental part, this paper not only carries out the comparative experiments of four different algorithms for the processing of stepped frequency multiresolution digital signal, but also simulates the convergence and convergence speed of the four different algorithms based on MATLAB and the corresponding modeling. The simulation and experimental frameworks are shown in the figure.

3.1. Principal Component Linear Prediction Processing Algorithm Based on Clutter Suppression Processing. At the clutter suppression level, this section is mainly based on the linear prediction of principal components for clutter suppression. At the level of principal component processing, it mainly analyzes and processes the collected echo signal from the perspective of two-dimensional determinant. At the level of principal component, the corresponding core processing ideas are as follows: based on the perspective of two-dimensional determinant analysis, it constructs and calculates the corresponding unrelated secondary fields, so as to analyze the relationship between the weights of the secondary fields. Taking the echo data as an example, the principal component analysis is carried out. Assuming that the corresponding echo data is Data1, the corresponding data is deconstructed based on the principal component. Formula (1) is the corresponding deconstruction formula. In the deconstruction formula, the corresponding mathematical symbol $n$ represents the label of the corresponding data sampling point, the mathematical symbol $m$ represents the number of data decomposition channels, and the corresponding $A i$ represents the $i$ th target vector.

$$
\text { Da } t a_{n}=A_{i-m} a_{i-m}^{T}=A_{1} a_{1}+A_{2} a_{2}+A_{3} a_{3}+\ldots A_{m} a_{m},
$$

Based on formula (1), the determinant of echo data is processed with covariance. The corresponding $q$ represents the projection size of the corresponding determinant in the corresponding direction after the corresponding echo data conversion, which represents the fluctuation degree of the echo data determinant in the corresponding direction and the corresponding energy size.

$$
\partial_{i}=\frac{s_{i}^{T} s_{i}}{m}, \quad i=1,2,3 \ldots m
$$

Based on this, the corresponding principal component processing algorithm mode is determined as shown in Figure 2. From the figure, we can see that the corresponding processing details are as follows.

Step 1: take the corresponding echo data as the corresponding processing object, and the corresponding processing matrix calculation formula is shown in formula (3), where the corresponding $n$ represents the corresponding echo data sampling points, and the corresponding $m$ represents the corresponding echo data number.

$$
Z_{i-m}=\left[\begin{array}{c}
s_{1} \\
s_{2} \\
\ldots \\
s_{n}
\end{array}\right]^{T}=\left[\begin{array}{cccc}
z_{11} & \ldots & \ldots & z_{1 m} \\
z_{21} & \ldots & \ldots & z_{2 m} \\
\ldots & \ldots & \ldots & \ldots \\
z_{m 1} & z_{m 2} & \ldots & z_{m m}
\end{array}\right],
$$

Step 2: use the traditional mean algorithm to process the echo data, and preprocess the corresponding echo data.

Step 3: based on the corresponding two-dimensional processing matrix, the corresponding echo data is processed by covariance matrix.

Step 4: analyze the corresponding eigenvalues and eigenvector values of the matrix after processing the corresponding echo data. 


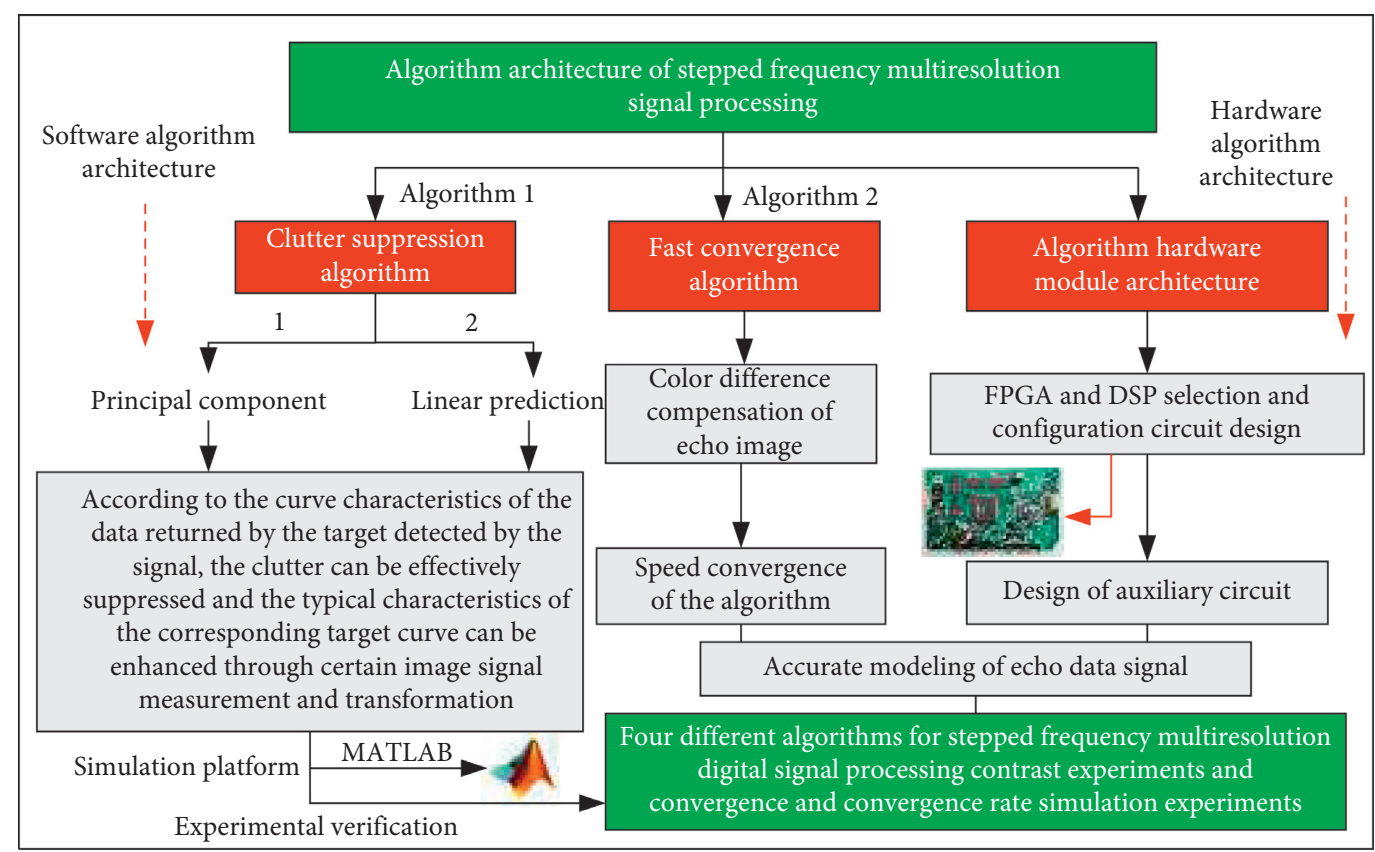

FIGURE 1: Architecture of stepped frequency multiresolution signal processing algorithm.

Echo data

input:Data1...Data $n$

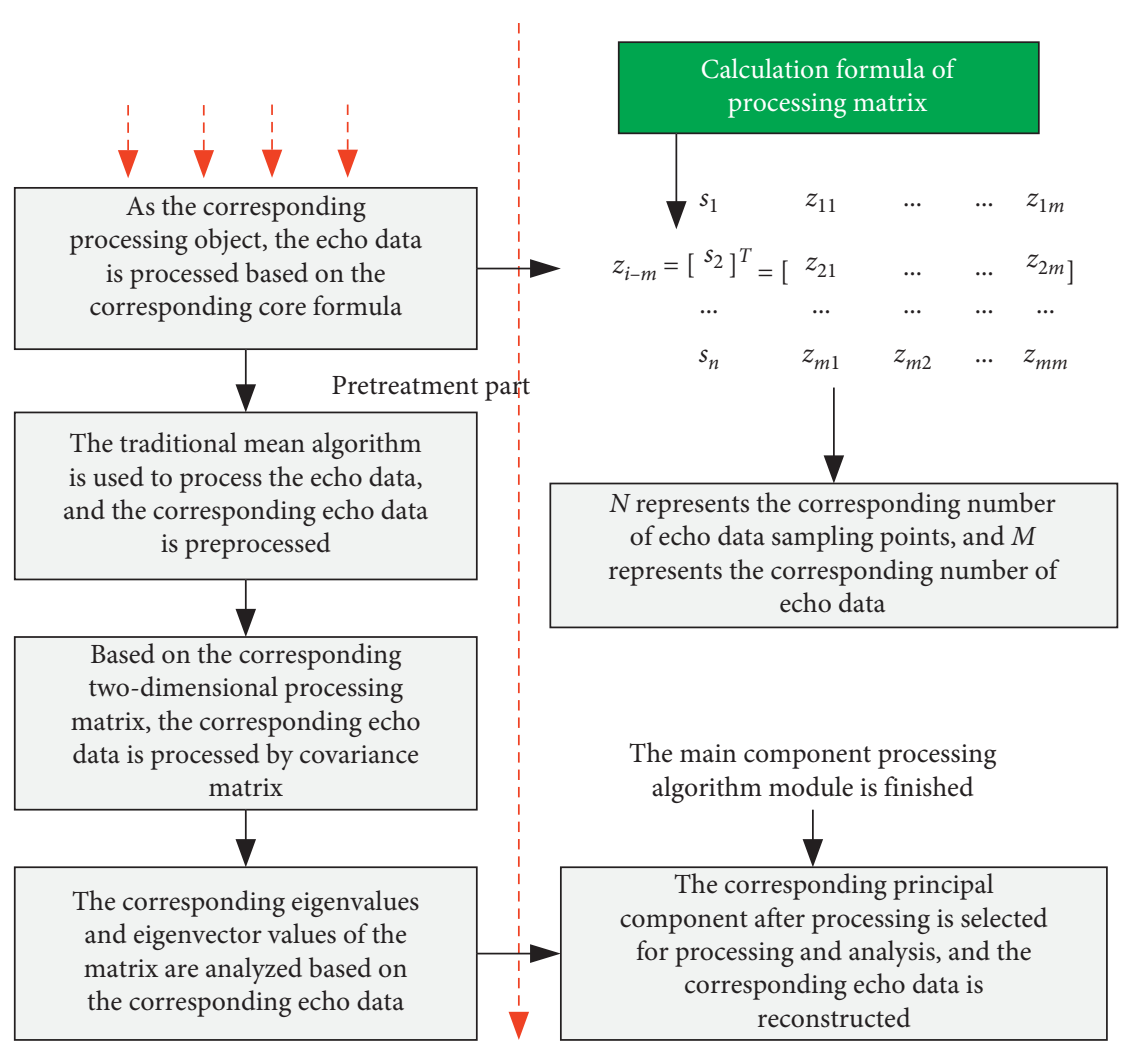

FIGURE 2: Algorithm operation flowchart of principal component processing algorithm module.

Step 5: select the corresponding principal component for processing and analysis, and reconstruct the corresponding echo data.
Based on the corresponding eigenvalues and eigenvector values obtained by the above principal component algorithm as the reference data of the linear prediction algorithm, the 
corresponding echo data is further processed and analyzed, which mainly realizes the prediction of the current measurement data. Based on this, the corresponding core processing formula is shown in formula (4).

$$
Z(i)=\left[\begin{array}{ccc}
a_{11} & \ldots & a_{1 m} \\
\ldots & \ldots & \ldots \\
a_{m 1} & \ldots & a_{m m}
\end{array}\right] *\left[\begin{array}{llll}
i & i-1 & \ldots & i-m
\end{array}\right] .
$$

For the complex interference factors faced by close range detection, the mathematical model is defined based on the above formula and the corresponding close range environmental factors, and the corresponding mathematical model is shown in Figure 3.

The echo data processed based on the principal component is taken as the corresponding reference data, and the corresponding bilateral data of the echo data matrix is selected as the linear prediction data of the current radar scan, and the number of corresponding reference data is set to $\mathrm{W}$. When the number of reference data is small, the corresponding reference range will narrow, and the corresponding prediction effect will be greatly reduced. When the number of reference data is large, the detection difference of the corresponding radar in the corresponding area will be large. In this way, the corresponding data will lose its representativeness. Therefore, when dealing with the number of reference data, this section filters based on the data eigenvalues after the principal component preprocessing, so as to solve the problem of the number of reference data. In this section, the actual selection is generally between 10 and 20. Based on the above principle analysis, the corresponding linear prediction algorithm flow based on principal component preprocessing is shown in Figure 4. From Figure 4, the corresponding processing steps are as follows.

Step 1: recombine the collected data after preprocessing the corresponding principal components, rename the corresponding data matrix, and reanalyze all the new matrices.

Step 2: select a reasonable subspace from the above analysis results and recombine the corresponding information data.

Step 3: conduct linear bilateral processing analysis on the data in step 2 above, and the number of corresponding linear bilateral processing data is set to 6 (12 in total).

Step 4: use the least square method to predict the above data, so as to finally obtain the measured value and the corresponding estimated value, and based on the measured value and the estimated value, reduce the difference.

In order to solve the coupling interference between the equipment antennas corresponding to the short-range detection system, eliminate the external interference factors of nonalgorithm factors, so as to further optimize the above algorithm; this section designs the auxiliary design of antenna coupling interference elimination. The coupling between antennas mainly exists in the fixed and stable clutter between the transmitting antenna and the receiving antenna. Based on the characteristics of this clutter, this section uses the air acquisition as the actual reference and subtracts the actual collected data. The corresponding processing steps are as follows.

Step 1: set up the corresponding short-range detection equipment, debug the corresponding parameters, and keep the interval between the receiving and transmitting antennas constant.

Step 2: shoot the corresponding equipment antenna to a clean environment without clutter interference, and collect data for the corresponding clean environment.

Step 3: preprocess the collected data to remove the interference of the corresponding target source. The collected echo data is mainly the mutual coupling direct wave between the receiving and transmitting antennas.

Step 4: average and store the corresponding data. The current reference data should be subtracted from the subsequent collected echo data so that the cleaner data can be obtained in the actual data processing of this algorithm.

3.2. Radius Pointing Transverse Mode Algorithm. In order to improve the convergence speed of the whole linear prediction algorithm and solve the unipolarity problem of the corresponding image problem, this section proposes the radius pointing transverse mode algorithm based on independent cell analysis algorithm. When the corresponding echo data is preprocessed by principal component, part of the signal is preprocessed at the same time. The corresponding transverse mode algorithm is used to iterate the corresponding data. The corresponding iteration termination condition is set as the absolute value threshold. Based on this, the inverse matrix of the corresponding echo data can be obtained. Based on the inverse matrix obtained above, the radius pointing transverse mode algorithm is used for depolarization multiplexing, so that the processing of echo data can get fast convergence. Based on this, the corresponding algorithm flow chart is shown in Figure 5. It can be seen from the figure that the corresponding algorithm flow details are as follows.

Step 1: preprocess and analyze the collected echo data. Step 2: based on constant modulus algorithm, the preprocessed data are processed iteratively.

Step 3: repeat step 2 until the iteration condition is terminated (a certain threshold is met) to obtain the corresponding inverse matrix of echo data.

Step 4: based on the inverse matrix of echo data, the radius pointing transverse mode algorithm is used for depolarization multiplexing to achieve fast convergence.

3.3. Algorithm Hardware System Design. The corresponding hardware system is designed based on the above algorithm, and the corresponding hardware system architecture is shown in Figure 6. It can be seen from the figure that the 


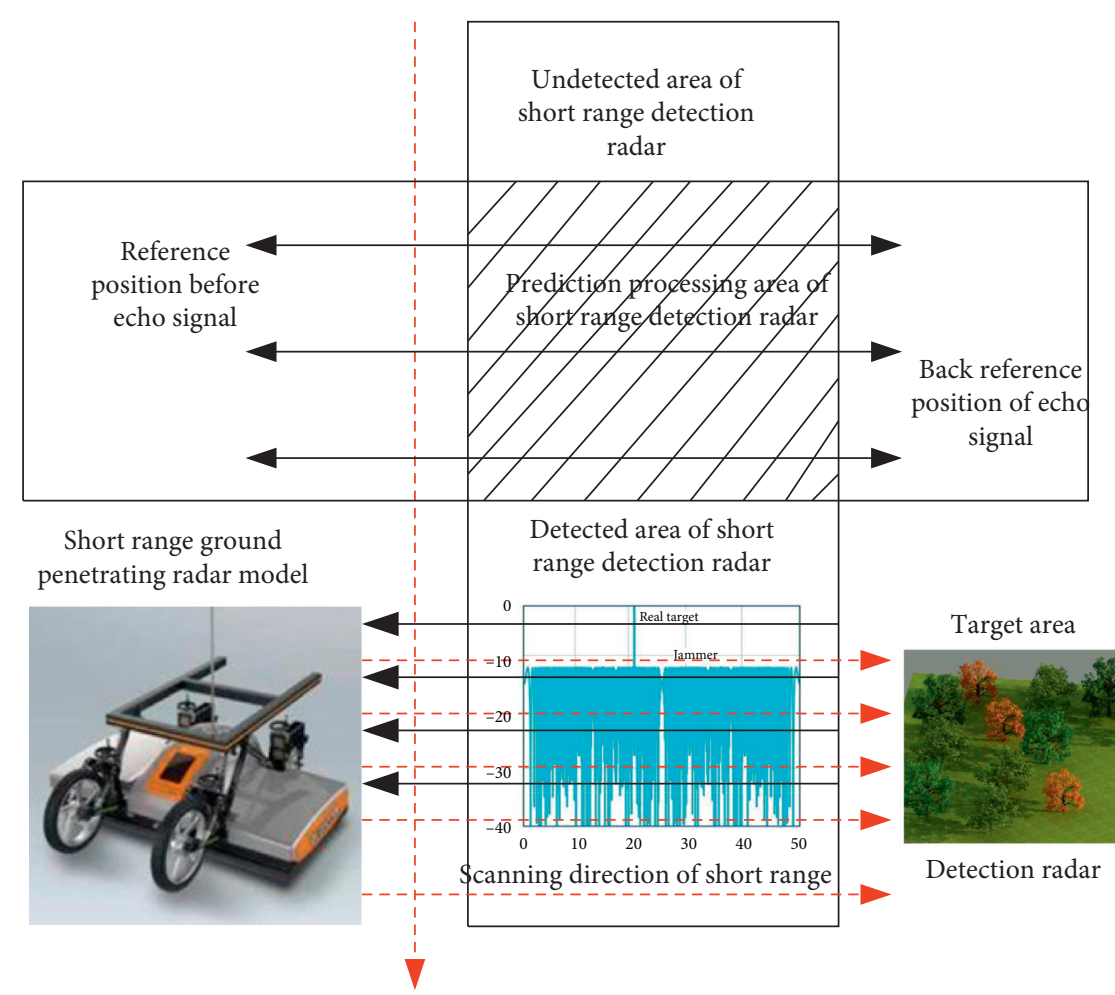

FIgURE 3: Mathematical model of linear prediction algorithm. processing algorithm (preprocessing)

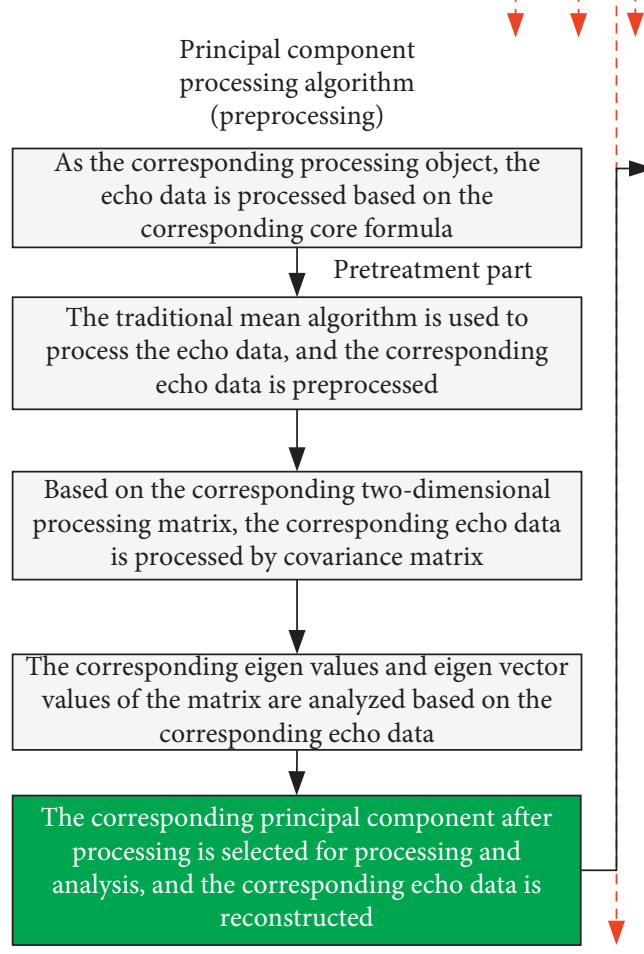
input:Data1...Data $n$ $\begin{array}{ccccc}1 & 1 & 1 & 1 & \text { Linear } \\ 1 & 1 & 1 & 1 & \end{array}$ †

After the preprocessed data of the corresponding principal components are recombined, the corresponding data matrix is renamed, and all the new matrices are analyzed by principal components

In the corresponding analysis results, the reasonable subspace is selected and the corresponding information data is recombined

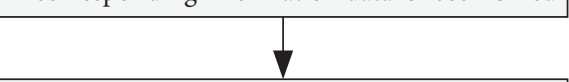

The data in the above step 2 is processed and analyzed by linear bilateral processing, and the number of corresponding linear bilateral processing data is set to 6 (12 in total)

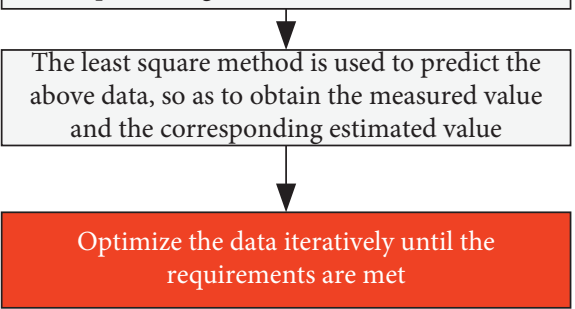

FIGURE 4: Algorithm operation flowchart of linear prediction processing algorithm module. 


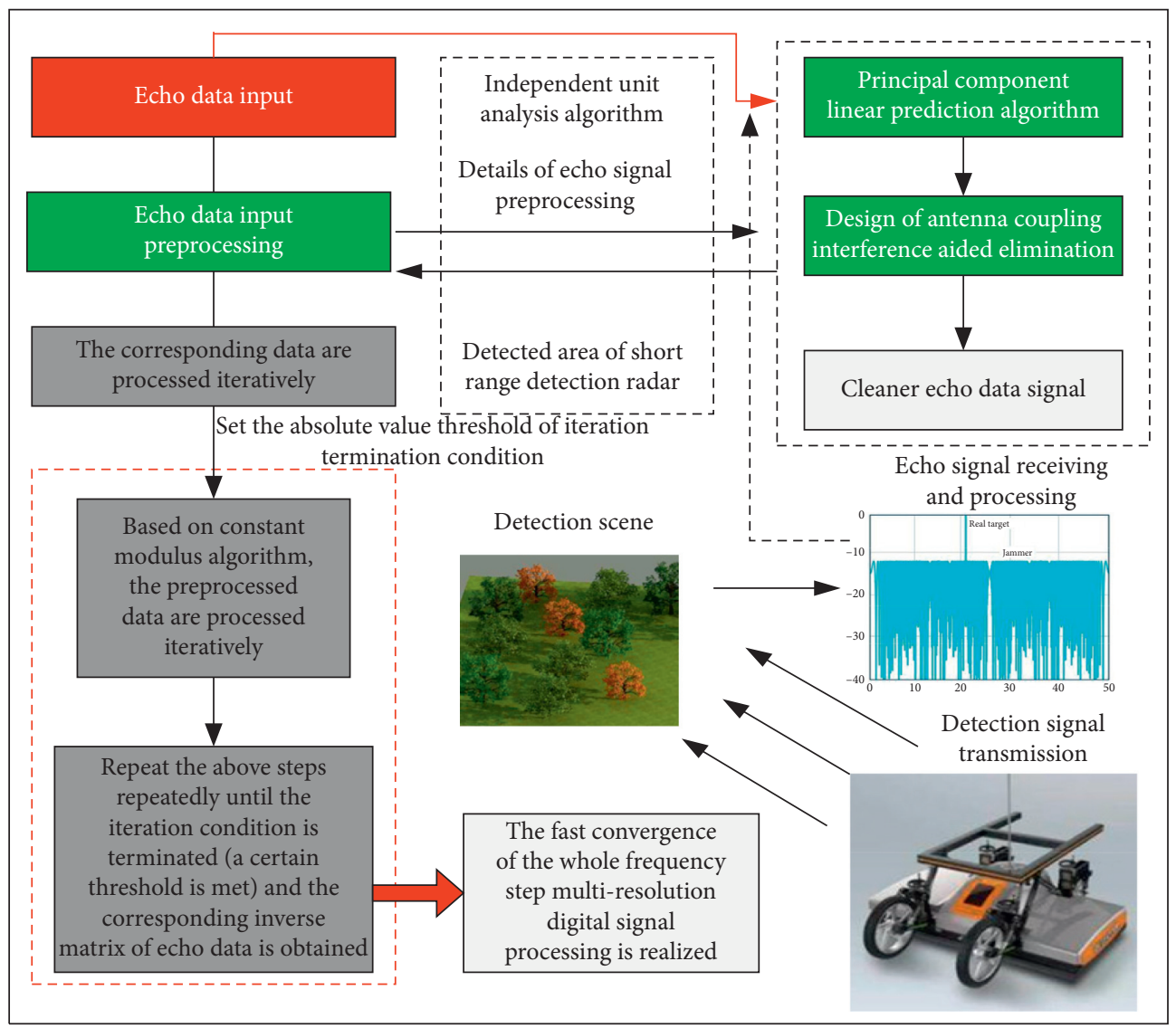

FIGURE 5: Flowchart of radius pointing transverse die algorithm.

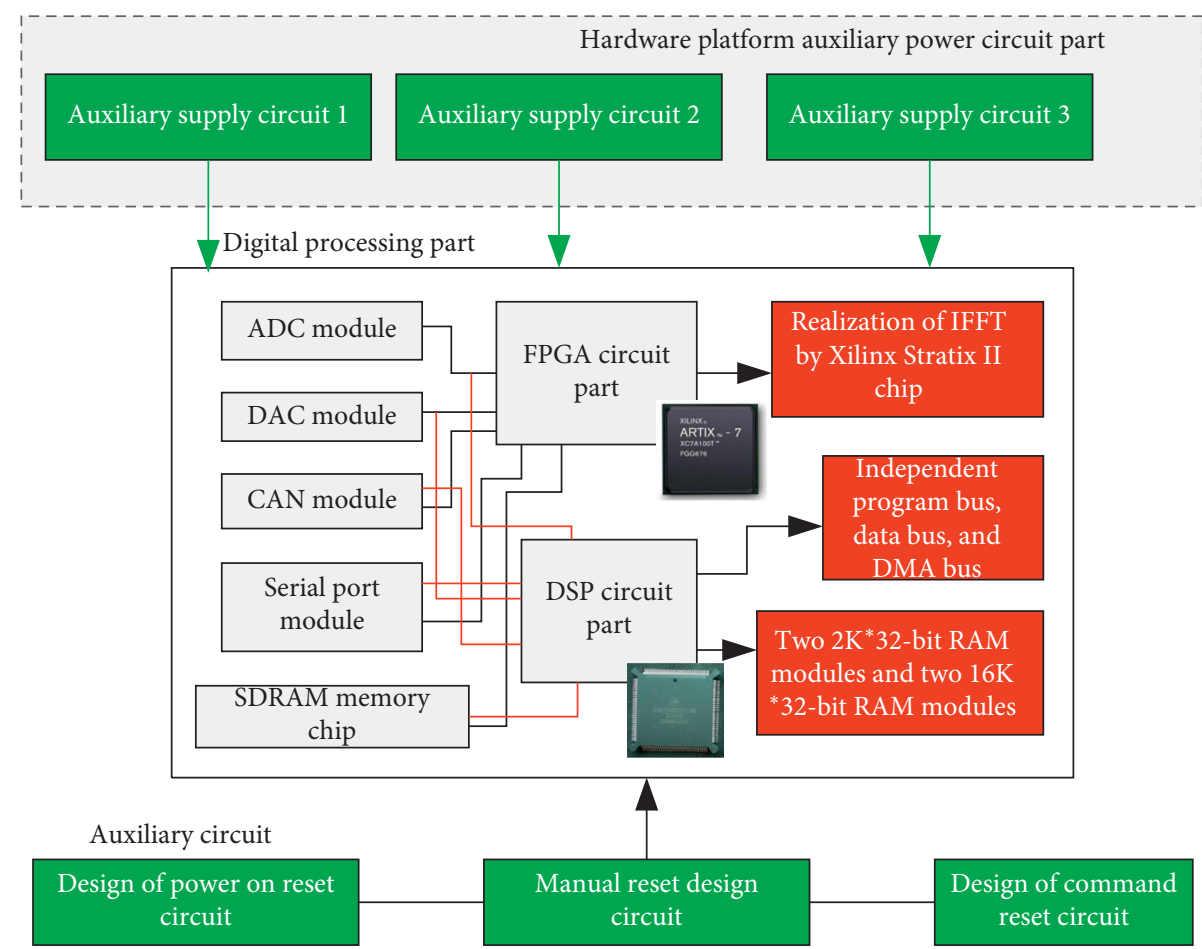

FIGURE 6: Hardware system architecture of stepped frequency multiresolution digital signal processing algorithm. 
corresponding hardware modules include FPGA data processing module, DSP data processing module, and auxiliary data processing module. The corresponding data processing module mainly uses the Xilinx chip for data processing. At the same time, the whole data processing unit is configured with two SDRAM memory chips and two flash chips. The corresponding front-end signal acquisition module mainly uses the multichannel ADC module and the corresponding FPGA interface module.

In the corresponding data acquisition level, the relevant chips of ad company are mainly used, and the main models include ad9042, ad9631, ad8138, and ad4938. For the circuit composed of ad9631 and its related resistance and capacitance, its main function is DC coupling circuit, and its corresponding circuit needs to be equipped with emitter follower composed of operational amplifier. Its main function is to draw out the internal bias voltage corresponding to ad9042, and the adjustment of bias voltage is still mainly carried out through operational amplifier. In the ADC system, bias correction is needed. The corrected bias voltage and the input echo digital signal are synthesized by the broadband low noise operational amplifier and used as the analog input of ad9042. In the design of the above data acquisition circuit, the power supply part of the digital circuit should be separated from the analog part to prevent its impact on the ADC conversion speed.

The implementation of IFFT in the key FPGA circuit of the corresponding digital processing part mainly realizes FFT operation through the IP core designed by Altera company. In the aspect of selecting the corresponding DSP, this paper mainly selects the TMS320C3X series of Texas Instruments. Its corresponding structure is relatively simple, and the corresponding peripheral devices are relatively few. At the same time, it has the advantages of high-quality floating-point, low power consumption, rich registers, and so on. At the same time, the corresponding hardware resources of the processor are also very rich. It has two $2 \mathrm{~K} * 32$ bit RAM modules and two $16 \mathrm{~K} * 32$-bit RAM modules. Its independent program bus, data bus, and DMA bus enable the processor to realize parallel processing.

In the above hardware architecture system, the corresponding auxiliary circuit design mainly includes power management circuit module, clock circuit design part, and reset circuit design part. The design of the corresponding power management module mainly considers the power supply to the corresponding FPGA and the corresponding DSP processor. In the aspect of clock design, the corresponding DSP, FPGA, $\mathrm{ADC}$, and all kinds of memory circuits in the hardware board are homologous with the external clock. Considering the selftest function of the whole system, crystal oscillator is set in the corresponding printed circuit board. The corresponding reset circuit design level mainly includes three types of reset design: power on reset design, manual reset design, and corresponding command reset design.

\section{Signal Modeling and Experimental Analysis}

4.1. Echo Signal Modeling. In order to better analyze the echo signal and prepare for the following experiments, this section will model and process the echo signal. The theoretical analysis shows that the echo signal is mainly composed of three parts: external interference clutter, external interference noise, and the corresponding short-range target signal. When the corresponding equipment collects the echo data signal, it can be directly reflected in the signal spectrum. According to practical experience, the corresponding clutter signal can be attributed to the clutter interference directly coupled from the near air or ground underground. To a certain extent, the modeling of echo signal can be understood as the modeling of clutter signal, which corresponds to the clutter in the near air, underground, and ground. It is mainly caused by the debris in the near environment. The characteristic of this kind of clutter signal is that its change in the corresponding amplitude point is not very large. The corresponding clutter hidden in the ground or in the air is closely related to the target signal source. The corresponding clutter is often caused by the serious distortion of the underground or near space environment or the uneven distribution of the media in the corresponding cavity. The corresponding clutter is a rapidly changing process. Therefore, the model corresponding to this kind of clutter is random. The signals generated by the corresponding target source are usually signals with small amplitude. The corresponding other types of clutter are usually random clutter, which are generally set as the standard Gaussian white noise mathematical model, and are basically processed and analyzed by this model in the actual model processing. The corresponding coupling signals between antennas are preprocessed by subtraction algorithm. Based on the above analysis, the corresponding mathematical model of echo signal is established as shown in the following formula:

$$
D=N_{1}+N_{2}+d N_{3} .
$$

The experimental environment is set as follows: the experimental data is collected by a research institute, the corresponding experimental environment is a close ground, and there are holes and small stones of different specifications on the corresponding ground. The experiment is based on the same hardware system, and the data is collected along the test site in the way of horizontal movement, and the corresponding moving speed is set to uniform. In the actual comparative experiment, the experimental condition variables of the two algorithms are kept unchanged. In experiment scenario 1, the signal-to-noise ratio (SNR) is introduced to distinguish the experimental results. As shown in Table 1, the SNR values of different algorithms are shown. From the table, it can be seen that the algorithm proposed in this paper has obvious advantages over the single mean algorithm, principal component algorithm, and linear prediction algorithm. It has higher clutter suppression effect and higher SNR in scenario 1.

In experiment scenario 2, metal interferences are added on the basis of experiment scenario 1, and the corresponding radar hardware equipment continuously detects the detected target for five times in the test area. The amplitude accumulation diagrams of corresponding different algorithms are shown in Figures 7(a)-7(d). It can be seen from the figure 
TABLE 1: Calculation results of SNR of different algorithms in scenario 1.

Digital signal processing algorithm

Signal-to-noise ratio

Raw data 1

$-15.345$

Mean method

8.987

Principal component algorithm

10.427

Linear prediction algorithm

15.293

An improved algorithm is proposed in this paper

30.114

Raw data 2

$-20.351$

Mean method

18.086

Principal component algorithm

Linear prediction algorithm

25.901

An improved algorithm is proposed in this paper

41.231

Raw data 3

Mean method

Principal component algorithm

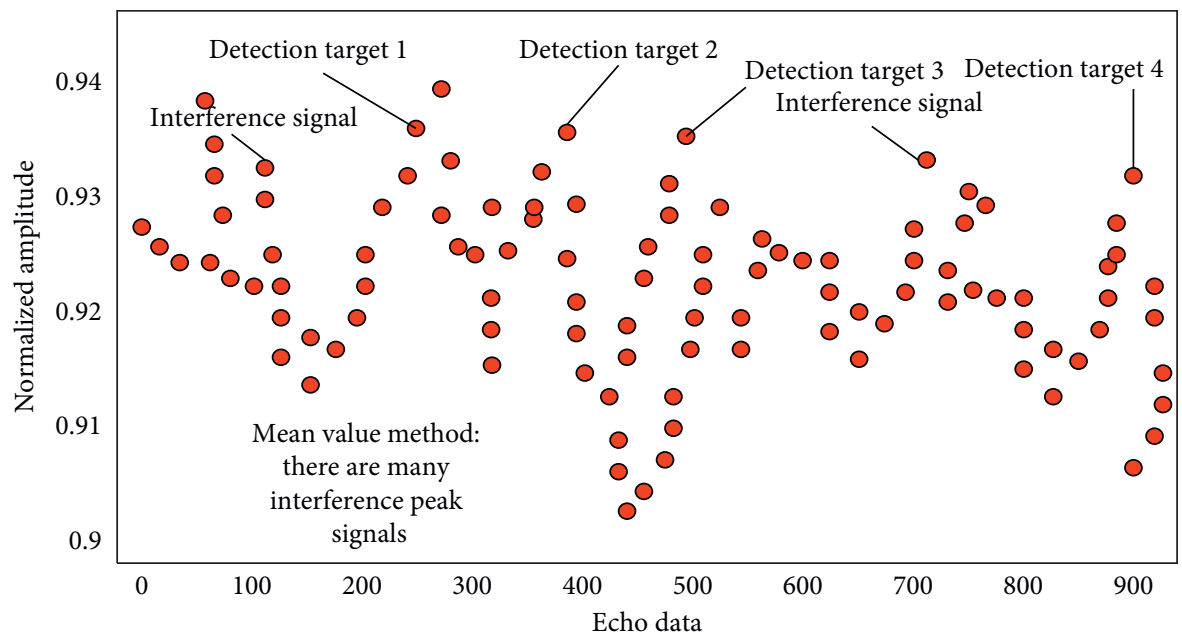

(a)

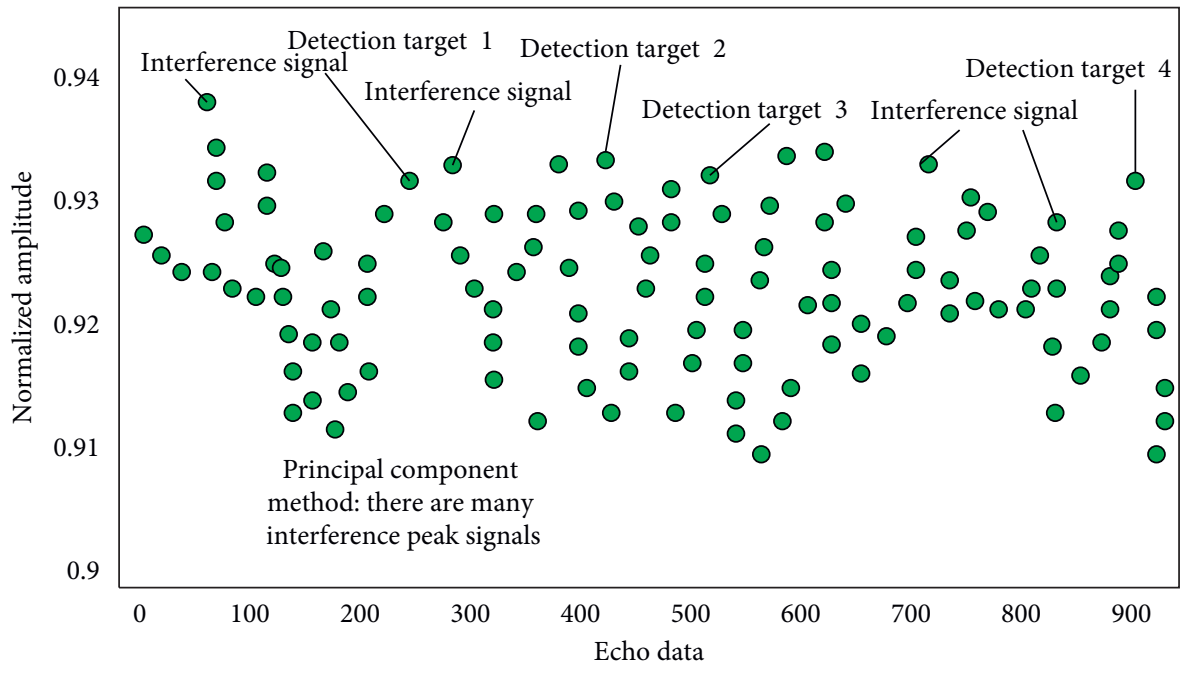

(b)

Figure 7: Continued. 


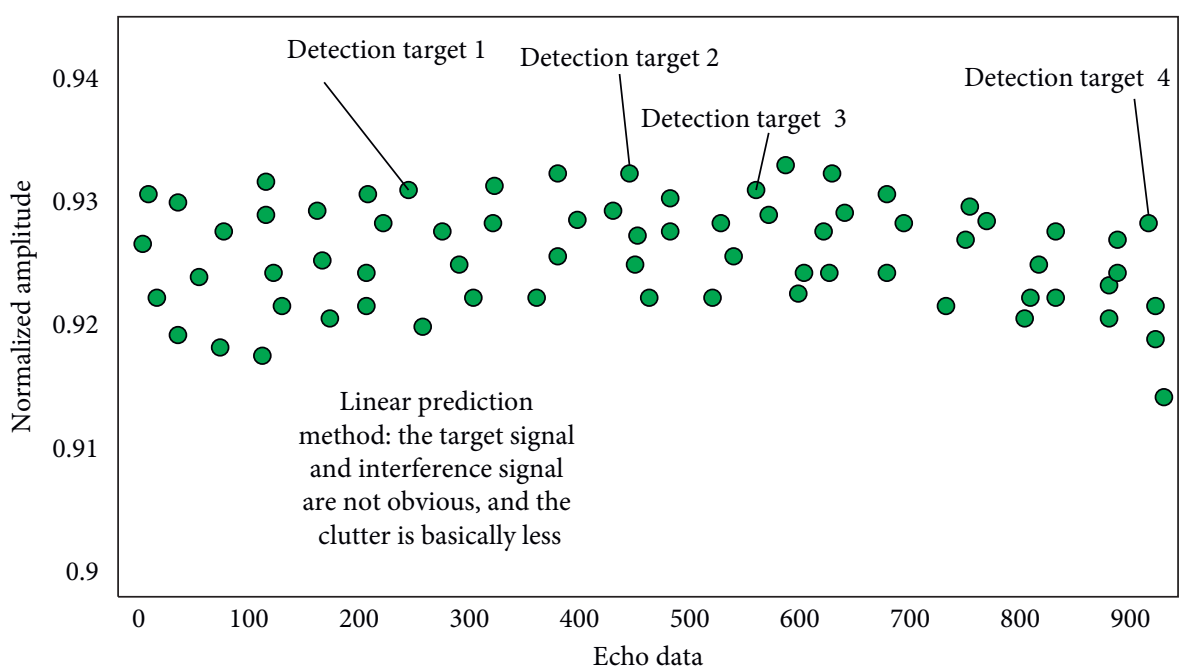

(c)

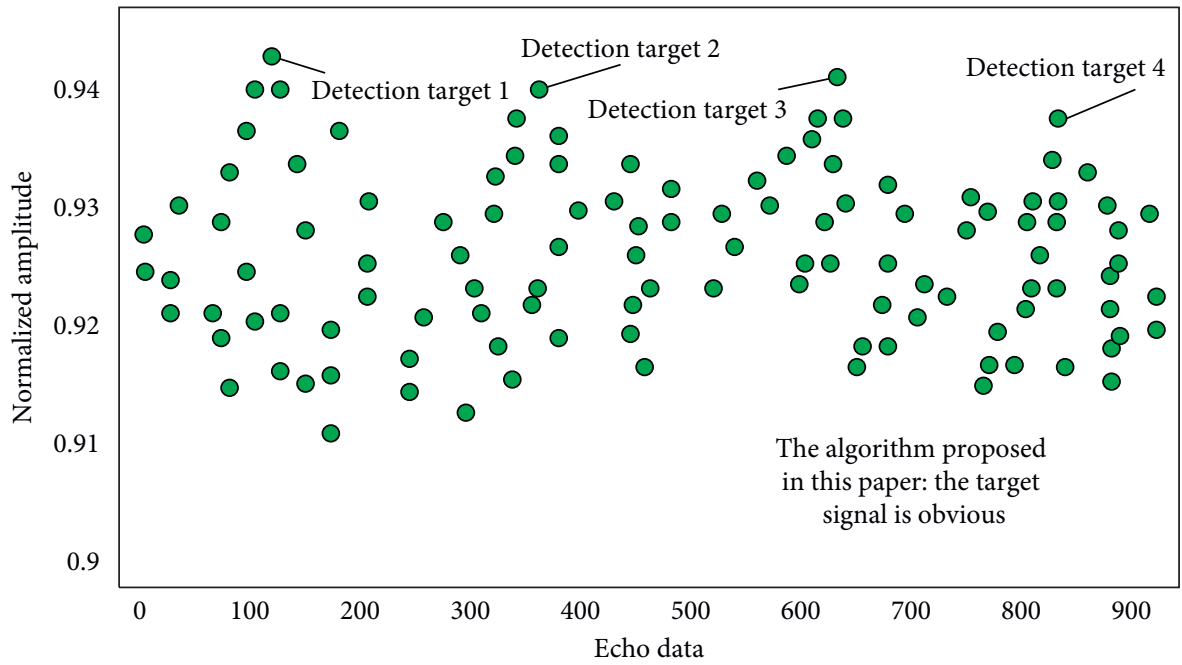

(d)

Figure 7: (a) Stacking chart of processing results under mean algorithm. (b) Stacking diagram of processing results under principal component algorithm. (c) Stacking chart of processing results under linear prediction algorithm. (d) The stack diagram of processing results under the algorithm proposed in this paper.

that compared with other algorithms, the algorithm proposed in this paper can obviously process five peak targets.

The SNR calculation table based on experimental scenario 2 is shown in Table 2 . It can be seen from the table that the algorithm proposed in this paper has obvious advantages over the single mean algorithm, principal component algorithm, and linear prediction algorithm. It has higher clutter suppression effect and higher SNR in scenario 2.

In order to verify the convergence rate of the algorithm, the simulation is carried out based on the simulation framework shown in Figure 8 below, and the corresponding simulation tool is MATLAB. The simulation architecture shown in the figure is used to simulate and analyze the convergence of different algorithms.

In the corresponding simulation architecture diagram, the simulation is mainly divided into four channels, corresponding to mean value method, principal component method, linear measurement method, and the algorithm proposed in this paper. In the actual simulation process, the principle of control variable method is followed, and the corresponding data source is guaranteed to be the same, and the corresponding data processing link is guaranteed to be the same. The only difference is the data processing module.

The convergence speed diagram of the four algorithms simulated based on the above simulation frame diagram is shown in Figure 9. From the figure, it can be seen that the convergence speed of the algorithm in this paper is faster than that of the other three algorithms. The algorithm proposed in this paper can reach the convergence point faster than the other three algorithms. At the same time, the curve trend in the graph can be seen. With the increase of data quantity, the convergence speed advantage of the proposed algorithm will be more obvious. 
TABLE 2: SNR calculation results of different algorithms in scenario 2.

Digital signal processing algorithm

Raw data 1

Mean method

Principal component algorithm

Linear prediction algorithm

An improved algorithm is proposed in this paper

Raw data 2

Mean method

Principal component algorithm

Linear prediction algorithm

An improved algorithm is proposed in this paper

Raw data 3

Mean method

Principal component algorithm

Linear prediction algorithm

An improved algorithm is proposed in this paper
Signal-to-noise ratio

$-11.547$

4.111

5.192

8.361

20.324

$-15.339$

8.774

15.934

20.331

25.143

$-20.302$

11.213

18.201

21.354

30.119

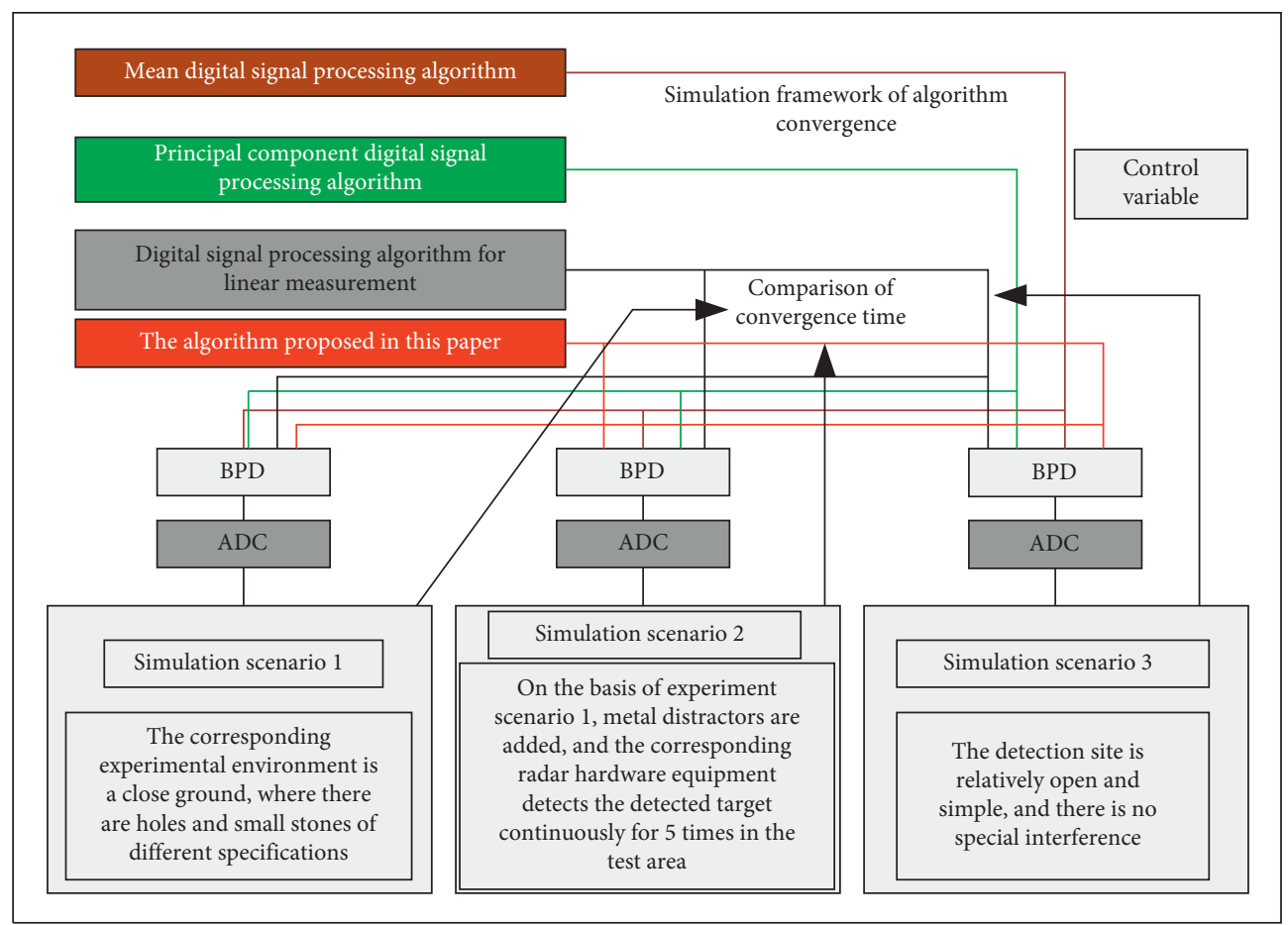

Figure 8: Simulation framework of algorithm convergence.

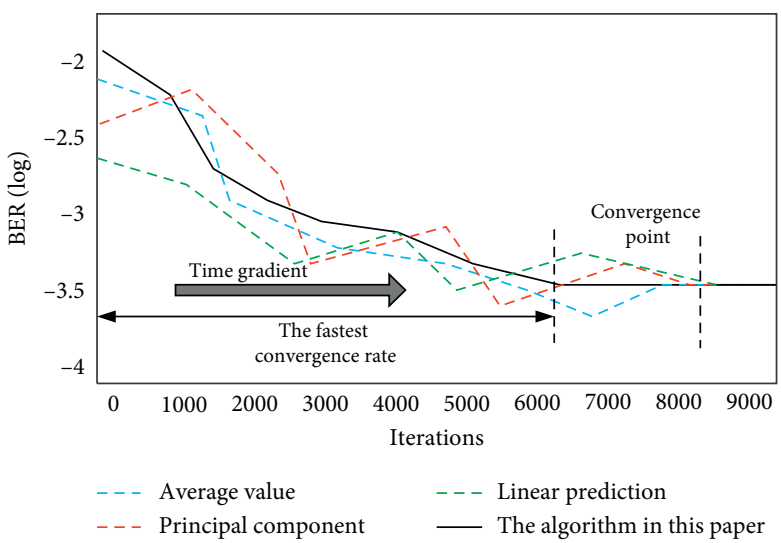

Figure 9: Curve of convergence rate of the algorithm. 
Based on the data results of scenario 1 and scenario 2, we can draw the following conclusions: the single mean algorithm, principal component analysis, and linear prediction algorithm can filter most of the clutter when removing the clutter of the echo signal, but it still has the situation that the clutter can not be filtered in the face of complex environment, and it is subject to the relatively large correlation level. Compared with the single algorithm mentioned above, the algorithm proposed in this paper has obvious clutter filtering advantages, and its corresponding convergence speed is also very fast. Therefore, based on the above experimental results, the proposed algorithm has obvious processing advantages and convergence advantages.

\section{Conclusions}

This paper mainly analyzes the current situation and disadvantages of stepped frequency multiresolution digital signal processing technology. Based on the traditional signal processing algorithm, this paper proposes a principal component linear prediction processing algorithm based on clutter suppression processing. According to the curve characteristics of the data returned by the target detected by the signal, through certain image signal measurement and transformation, the clutter can be effectively suppressed and the typical characteristics of the corresponding target curve can be enhanced. For the convergence problem of signal processing and the corresponding image chromatic aberration compensation problem, this paper realizes the chromatic aberration compensation of the corresponding target echo image based on the radial pointing transverse mode algorithm and enhances the convergence speed of the whole algorithm system. In the experimental part, the proposed optimization algorithm is compared with the traditional algorithm. The experimental results show that the proposed algorithm has obvious advantages in the convergence of signal processing and antijamming performance and has the promotion value. This paper will focus on the optimization of convergence and antijamming for largescale echo data.

\section{Data Availability}

No data were used to support the findings of the study.

\section{Conflicts of Interest}

The author declares that there are no conflicts of interest.

\section{Acknowledgments}

The author acknowledges the scientific research projects of Xi'an Peihua University in 2020, Design of Wearable Motion Data Display System Based on Bluetooth (PHKT2029).

\section{References}

[1] H. Iker and C. Zdemir, "Adaptation of stepped frequency continuous waveform to range-Doppler algorithm for SAR signal processing," Digital Signal Processing, vol. 106, no. 4, p. $102826,2020$.

[2] C. L. Rino, K. M. Groves, C. S. Carrano et al., "Digital signal processing for ionospheric propagation diagnostics," Radio Science, vol. 50, no. 8, pp. 11-23, 2015.

[3] S. Li, M. Xue, T. Qing, C. Yu, L. Wu, and S. Pan, "Ultrafast and ultrahigh-resolution optical vector analysis using linearly frequency-modulated waveform and dechirp processing," Optics Letters, vol. 44, no. 13, p. 3322, 2019.

[4] S. J. Tingay, P. J. Hancock, R. B. Wayth et al., "A multiresolution, multi-epoch low radio frequency survey of the Kepler K2 mission Campaign 1 field," The Astronomical Journal, vol. 152, no. 4, pp. 112-121, 2016.

[5] O. Lobachev, C. Ulrich, B. S. Steiniger, V. Wilhelmi, V. Stachniss, and M. Guthe, "Feature-based multi-resolution registration of immunostained serial sections," Medical Image Analysis, vol. 35, no. 4, pp. 288-302, 2017.

[6] M. Chagnon, M. Morsy-Osman, D. Patel, V. Veerasubramanian, A. Samani, and D. Plant, "Digital signal processing for dual-polarization intensity and interpolarization phase modulation formats using Stokes detection," Journal of Lightwave Technology, vol. 34, no. 1, pp. 188-195, 2016.

[7] R. Kuldvee, M. Kaljurand, and H. C. Smit, "Improvement of signal-to-noise ratio of electropherograms and analysis reproducibility with digital signal processing and multiple injections," Journal of Separation Science, vol. 21, no. 3, pp. 169-174, 1998.

[8] M. Agostini, M. Allardt, A. M. Bakalyarov et al., "Improvement of the energy resolution via an optimized digital signal processing in GERDA Phase I," European Physical Journal C, vol. 75, no. 6, p. 255, 2015.

[9] S. Schaefer, M. Gregory, and W. Rosenkranz, "Coherent receiver design based on digital signal processing in optical high-speed intersatellite links with M -phase-shift keying," Optical Engineering, vol. 55, no. 11, p. 111614, 2016.

[10] Y. Zhao, B. Hu, Z. A. He et al., "Generation and coherent detection of QPSK signal using a novel method of digital signal processing," Modern Physics Letters B, vol. 32, no. 4, p. 1850103, 2018.

[11] J. Yu, L. Wang, P. Liao et al., "Method of digital signal process aided by control signal for burst-mode coherent receivers," Optical Engineering, vol. 54, no. 11, p. 116103, 2015.

[12] W. Nascimento Lopes, F. Isaac Ferreira, F. Aparecido Alexandre et al., "Digital signal processing of acoustic emission signals using power spectral density and counts statistic applied to single-point dressing operation," IET Science, Measurement \& Technology, vol. 11, no. 5, pp. 631-636, 2017.

[13] M. Mazur, J. Schröder, A. Lorences-Riesgo, T. Yoshida, M. Karlsson, and P. A. Andrekson, "Overhead-optimization of pilot-based digital signal processing for flexible high spectral efficiency transmission," Optics Express, vol. 27, no. 17 , p. $24654,2019$.

[14] T. Yellamraju, A. J. Magana, and M. Boutin, "Investigating students' habits of mind in a course on digital signal processing," IEEE Transactions on Education, vol. 62, no. 4, pp. 312-324, 2019.

[15] M. Altman, "Ardutouch: an Arduino-compatable synthesizer: digital signal processing squeezed into an easy-to-build kit[Resources_Hands on]," IEEE Spectrum, vol. 55, no. 12, pp. 21-22, 2018.

[16] K. Zhang, Q. Zhuge, H. Xin, and W. Hu, "Performance comparison of DML, EML and MZM in dispersion-unmanaged short reach transmissions with digital signal 
processing," Optics Express, vol. 26, no. 26, pp. 34288-34304, 2018.

[17] K. V. Ajay, A. Navneet, and I. Arif, "Comparison of digital signal processing, feedback and feedforward compensation technique for dual polarization 128-QAM radio over fiber link," Optik, vol. 174, no. 5, pp. 68-76, 2018.

[18] R. Asif, R. Ahmad, and R. Basir, "Hybrid opto-digital signal processing in $112 \mathrm{Gbit} / \mathrm{s}$ DP-16QAM and DP-QDB transmission for long-haul large- $\$ \$ \backslash$ hbox $\{\mathrm{A}\}_{-} \mid$mathrm $\{$eff $\} \$ A$ eff pure-silica-core fiber links," Photonic Network Communications, vol. 32, no. 1, pp. 1-8, 2016.

[19] Z. Wang, J. Zhang, N. Verma et al., "Reducing quantization errors for inner-product operations in embedded digital signal processing systems [Tips\&Tricks]," IEEE Signal Processing Magazine, vol. 33, no. 6, pp. 141-147, 2016.

[20] Y. Liu, H. Xiong, C. Dong, C. Zhao, Q. Zhou, and S. Li, "Realtime signal processing in field programmable gate array based digital gamma-ray spectrometer," Review of Scientific Instruments, vol. 91, no. 10, p. 104707, 2020.

[21] W. Wang, H. Li, P. Zhao et al., "Advanced digital signal processing for reach extension and performance enhancement of 112 gbps and beyond direct detected DML-based transmission," Journal of Lightwave Technology, vol. 37, no. 1, pp. 163-169, 2018.

[22] P. S. Lee, C. S. Lee, and J. H. Lee, "Development of FPGAbased digital signal processing system for radiation spectroscopy," Radiation Measurements, vol. 48, no. 4, pp. 12-17, 2013.

[23] Q. Zhuge, M. Morsy-Osman, M. Chagnon, X. Xu, M. Qiu, and D. V. Plant, "Terabit bandwidth-adaptive transmission using low-complexity format-transparent digital signal processing," Optics Express, vol. 22, no. 3, p. 2278, 2014.

[24] X. Liu, S. Chandrasekhar, and P. J. Winzer, "Digital signal processing techniques enabling multi- $\mathrm{Tb} \backslash / \mathrm{s}$ superchannel transmission: an overview of recent advances in DSP-enabled superchannels," IEEE Signal Processing Magazine, vol. 31, no. 2, pp. 16-24, 2014. 